\title{
Heterocyclic synthesis with activated nitriles : an expeditus synthetic approach to polyfunctionally substituted pyrroles, heterocyclopyrimidines and coumarins
}

\author{
Ayman W. Erian, ${ }^{\mathrm{a} *}$ Sherif M. Sherif ${ }^{\mathrm{a}}$, and Nadia R. Mohamed ${ }^{\mathrm{b}}$ \\ ${ }^{a}$ Department of Chemistry, Faculty of Science, Cairo University, Giza, Egypt \\ ${ }^{b}$ Department of Photochemistry, National Research Center, Dokki, Giza, Egypt \\ E-mail: erian10@yahoo.com
}

(received 12 Jun 01; accepted 07 Apr 02; published on the web 15 Apr 02)

\begin{abstract}
The applicability and synthetic potency of the new reagent $N-(2,2-$ dicyanoethenyl)aminoacetonitrile (3) to develop an expeditious convenient synthetic route of unique polyfunctionally substituted pyrroles, heterocyclopyrimidines and $2 \mathrm{H}$-1-benzopyran-2ones is reported. Chemical and spectroscopic evidence for the structures of the newly synthesized compounds are described.
\end{abstract}

Keywords : Nitriles, pyrroles, pyrimidines, coumarins

$\alpha, \beta-\pi$-Deficient nitrile reagents are highly reactive and are extensively utilized as unique synthons for the construction of a variety of unique heterocyclic ring systems. ${ }^{1-5}$ In continuation with our medicinal chemistry program directed towards the development of new procedures for the synthesis of azoles, azines and their condensed derivatives utilizing readily obtainable polyfunctional nitriles, ${ }^{6-10}$ we report herein, an easy, facile and new synthetic methodology for the synthesis of $N$-(2,2-dicyanoethenyl)aminoacetonitrile (3). The latter proved to be a new key precursor for the synthesis of some polyfunctionally substituted pyrroles, thiazolo[1,2a]pyrimidines, pyrido[1,2-a]pyrimidines, and coumarins. The importance of those compounds is due to their diverse potential biological and physiological expected broad spectrum. ${ }^{11-14}$

\section{Results and Discussion}

The key precursor $N$-(2,2-dicyanoethenyl)aminoacetonitrile (3) was prepared, in a facile high yield reaction, upon treatment of aminoacetonitrile hydrogen sulphate (1) with an equimolar amount of ethyl 2-cyano-3-ethoxypropenonitrile (2) in an aqueous alcoholic $\mathrm{NaOH}$ solution at 
room temperature. Assignment of structure 3 for the reaction product was based on its correct elemental analyses and compatible spectroscopic data. Thus, its mass spectrum revealed a molecular ion peak at $m / z(\%)=132(18 \%)$ corresponding to the molecular formula $\mathrm{C}_{6} \mathrm{H}_{4} \mathrm{~N}_{4}$. Its IR spectrum showed absorption peaks at $v 3340(\mathrm{NH})$, and 2220, 2216, $2208 \mathrm{~cm}^{-1}(3 \mathrm{CN})$. Its ${ }^{1} \mathrm{H}-$ NMR spectrum (DMSO- $d_{6}$ ) showed a singlet signal $(2 \mathrm{H})$ at $\delta 4.12 \mathrm{ppm}$ assigned for the $\mathrm{CH}_{2}$ protons, a singlet at $\delta 7.32 \mathrm{ppm}$ assigned for the $\mathrm{CH}$ proton and a broad singal at $\delta .9 .11 \mathrm{ppm}$ assigned for the $\mathrm{NH}$ proton which underwent a facile hydrogen deuterium exchange and disappeared upon addition of $\mathrm{D}_{2} \mathrm{O}$ to the NMR sample. The structure of $\mathbf{3}$ was further confirmed on the basis of its chemical behaviour towards different chemical reagents.

Compound 3 reacted with an equimolar amount of diazomethane in dry ether at ice bath to yield the corresponding $N$-methyl derivative $\mathbf{4}$. The active methylene group in compound 4 underwent an electrophilic substitution upon coupling with equimolar amounts of aryldiazonium chloride salts to yield the corresponding hydrazone coupling products $\mathbf{5 a} \mathbf{a} \mathbf{b}$. Compound $\mathbf{5}$ proved to exist predominantly in the hydrazone form rather than the azo form on the basis of ${ }^{1} \mathrm{H}-\mathrm{NMR}$ data. Thus, e.g. 5a revealed, besides the expected signals, the presence of a singlet signal exchangeable with $\mathrm{D}_{2} \mathrm{O}$ at $\delta 8.21 \mathrm{ppm}$ attributed to the hydrazone $\mathrm{NH}$ function. Furthermore, its UV spectrum showed absorption maxima at $\lambda 420(\varepsilon=35280)$ and $330 \mathrm{~nm}(\varepsilon=23470)$ in accordance with those for hydrazo functions which are reported to exhibit strong absorption at wavelengths higher than $310 \mathrm{~nm} .^{15,16}$

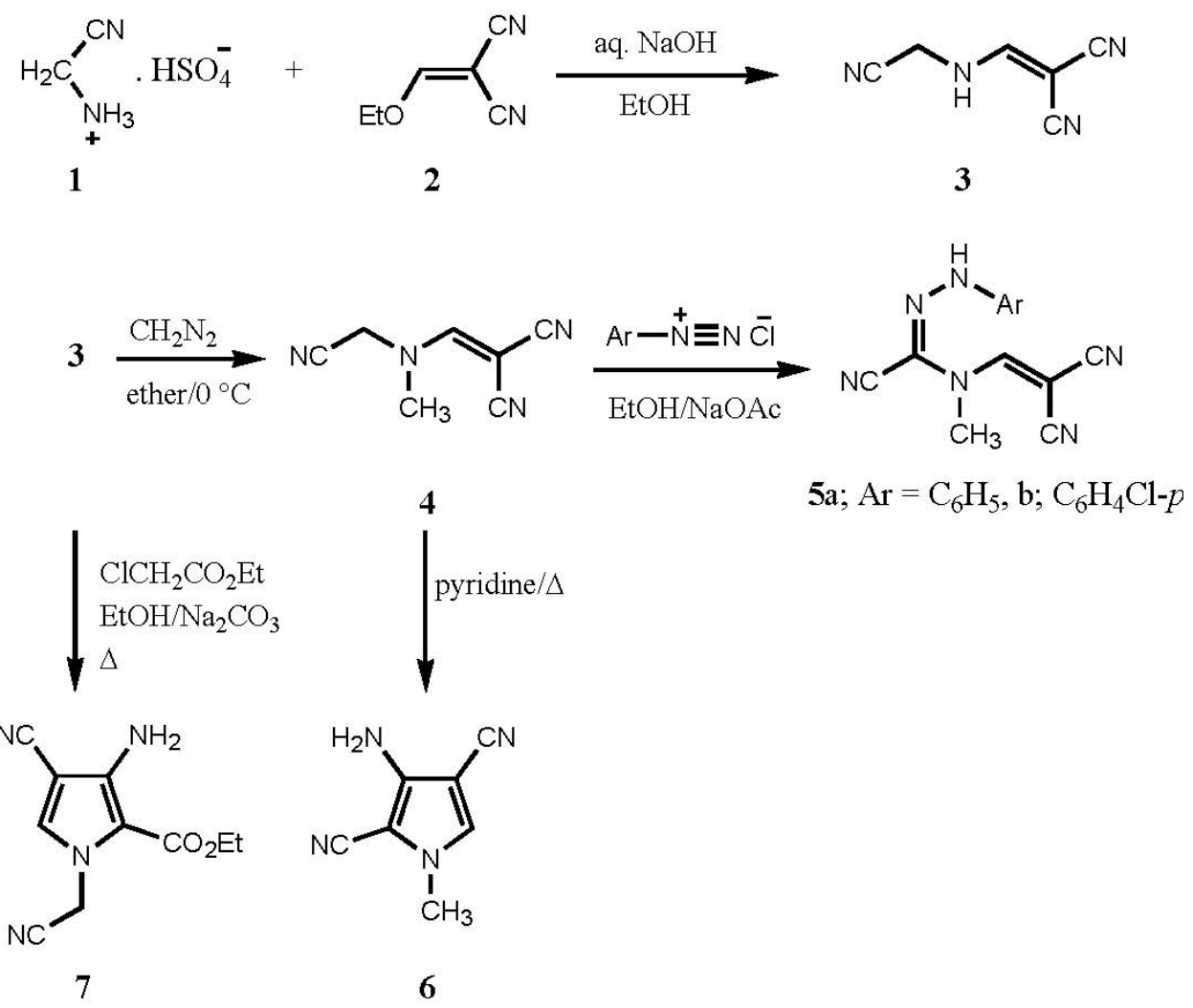

\section{Scheme 1}


Compound 4 underwent an intramolecular heterocyclization, upon boiling under reflux in dry pyridine, to afford 3-amino-2,4-dicyano-1-methylpyrrole (6). Compound 3 readily reacted with an equimolar amount of ethyl chloroacetate in aqueous $\mathrm{Na}_{2} \mathrm{CO}_{3}$ solution, under reflux, to yield ethyl 3-amino-4-cyano-1-cyanomethylpyrrole-2-carboxylate (7) (Scheme 1). Formation of 7 is assumed to proceed via an initial $\mathrm{N}$-alkylation and a subsequent intramolecular heterocyclization via a Michael-type nucleophilic addition of the $\mathrm{CH}_{2}$ protons to the cyano function.

The base-promoted nucleophilic addition of phenyl isothiocyanate to $\mathbf{3}$ has been achieved, upon boiling under reflux in an aqueous ethanolic $\mathrm{NaOH}$ solution, to yield the corresponding 1cyanomethyl-4-oxo-2-thioxopyrimidine derivative 8 (Scheme 2). The reaction apparently involves an intramolecular heterocyclization via the addition of the amine nitrogen of $\mathbf{3}$ to the carbon of isothiocyanate function and a subsequent alkaline hydrolysis of the imino function under the reaction conditions.17 Reaction of 8 with an equimolar amount of $N, N-$ dimethylformamide dimethylacetal (DMF-DMA) in dry dioxane, under reflux, furnished 5cyano-1-[1'-cyano-2'-dimethylaminoethenyl)-4-oxo-3-phenyl-1,2,3,4-tetra-hydro-2-

thioxopyrimidine (9) in an acceptable yield. Treatment of 9 with an equimolar amount of hydrazine hydrate in refluxing ethanol furnished exclusively a single product that could be formulated as 1-[3'-amino-pyrazol-4'-yl]-5-cyano-4-oxo-3-phenyl-1,2,3,4-tetrahydro-2thioxopyri-midine (10). Characterization of $\mathbf{1 0}$ is consistent with its elemental analysis and spectral data. However, compound $\mathbf{1 0}$ was an unstable yellow product that decomposed automatically at $25{ }^{\circ} \mathrm{C}$ within two days, therefore, it should be stored in a refrigerator. When compound 3 was treated with an equimolar amount of $N, N$-dimethylformamide dimethylacetal (DMF-DMA) in dry dioxane, under reflux, the corresponding 2,2-dimethylaminoethenyl derivative 11 was isolated. Elucidation of the proposed structure 11 was based on its correct elemental analyses and compatible spectroscopic data. 


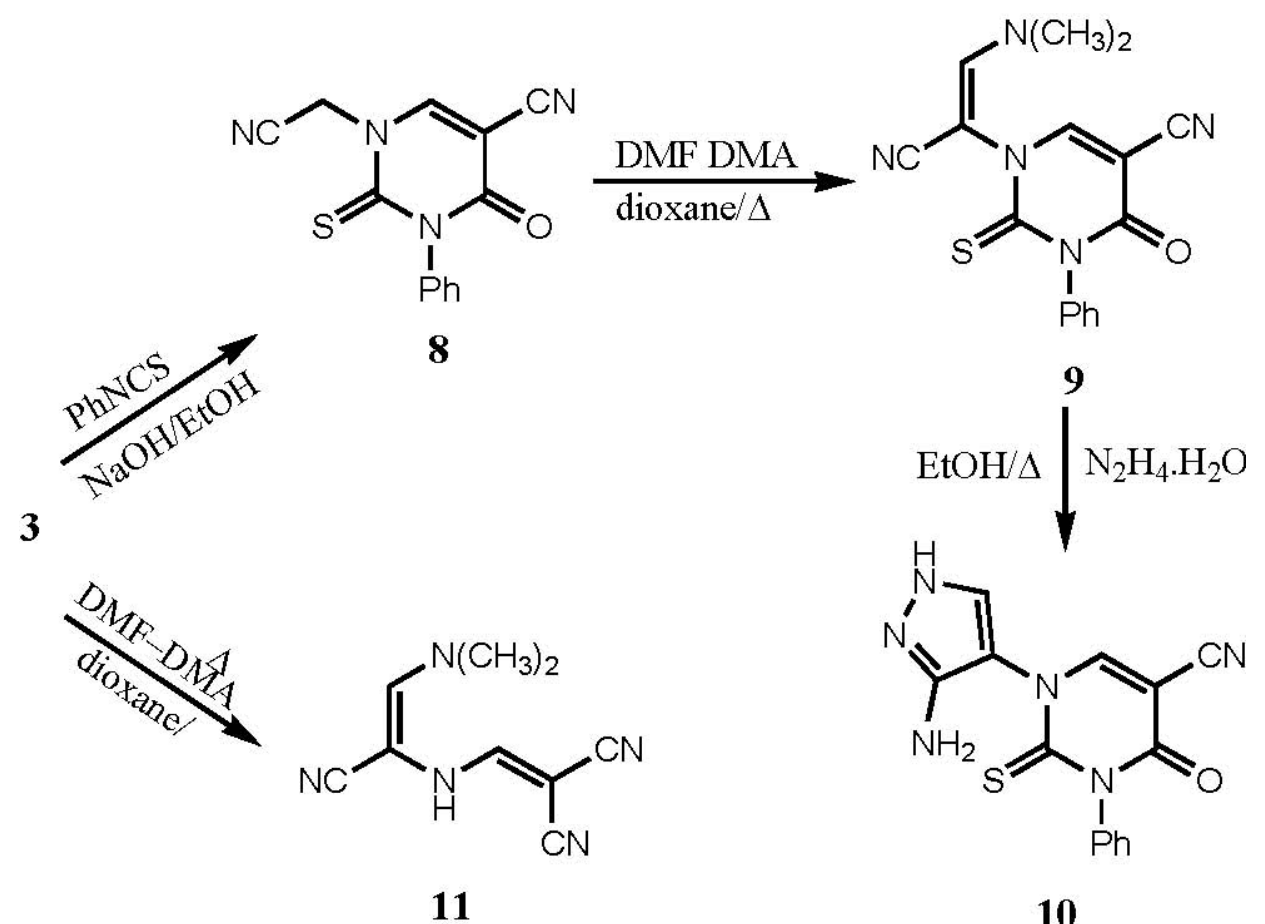

Scheme 2

Next, it was of interest to explore the scope, limitations and generality of $\mathbf{1 1}$ as a precursor for the synthesis of some difficult to access polyfunctionally substituted fused pyrimidine or benzopyranone derivatives for which we might expect a wide spectrum of bioresponses. Thus, reaction of $\mathbf{1 1}$ with equimolar amounts of $\mathrm{N}$-nucleophiles; namely, 2-aminopyridine or 2-aminothiazole, upon reflux in glacial acetic acid, afforded the corresponding 4H-pyrido[1,2a]pyrimidin-4-one $\mathbf{1 3}$ and 5H-thiazolo[1,2-a]pyrimidin-5-one $\mathbf{1 5}$ derivatives, respectively, in reasonable yields (Scheme 3). The identity of the product was established on the basis of elemental analyses and spectral background in each case. 


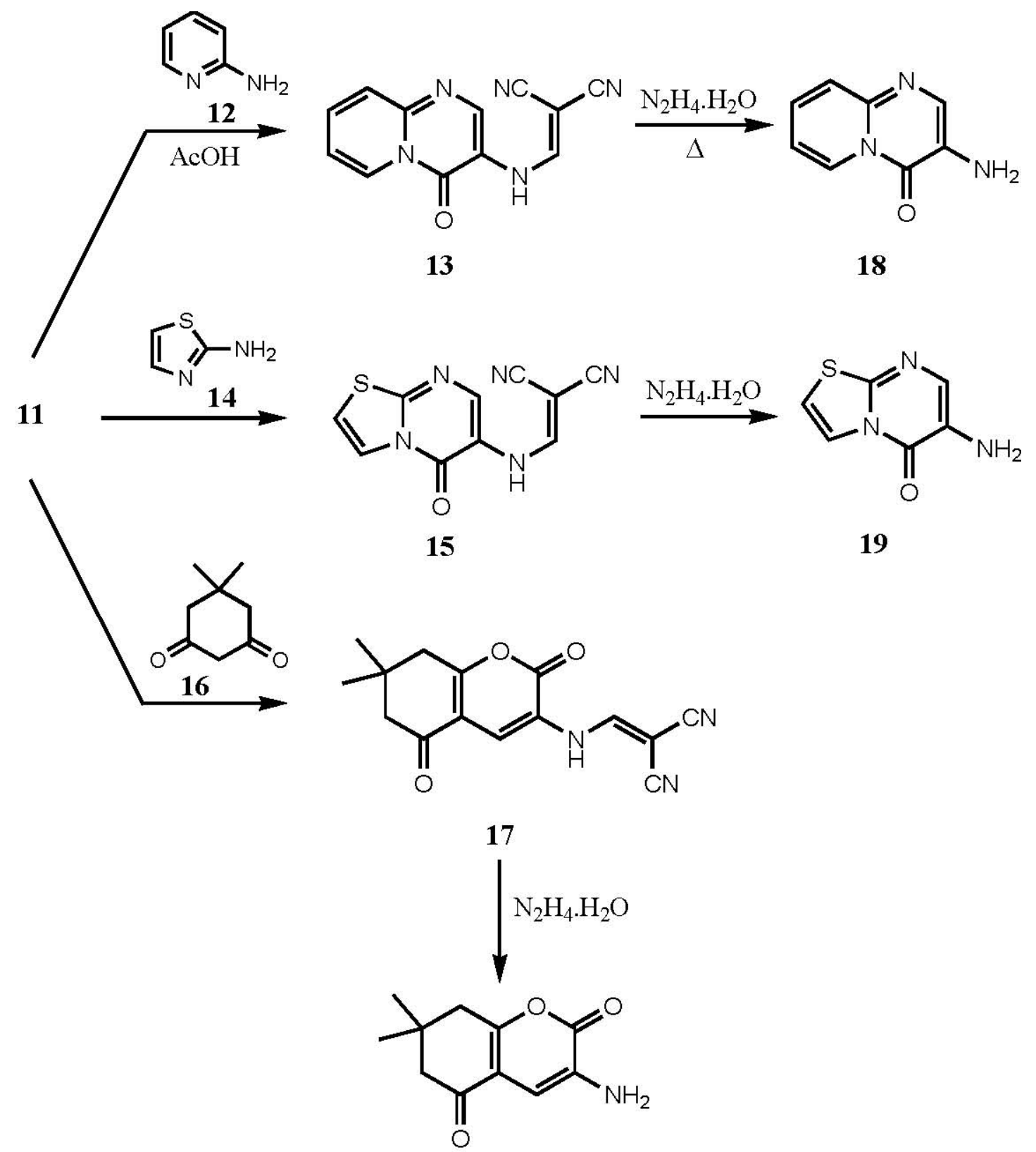

20

\section{Scheme 3}

Analogously, compound $\mathbf{1 1}$ reacted with $C$-nucleophiles e.g. dimedone, under the same experimental conditions to yield the corresponding 5,6,7,8-tetrahydro-2H-1-benzopyran-2-one derivative 17.

The free amino heterocycles 18-20 could be achieved on treatment of the appropriate 2,2dicyanoethenylamino heterocycles 13, 15, or 17 with hydrazine hydrate in ethanol at reflux temperature, respectively.

In conclusion, the results presented in this article, indirectly extend and broaden the 
knowledge in the area of activated nitriles and demonstrate a general applicable methodology for the construction of a wide variety of polyfunctionally substituted annelated pyrimidines and $2 \mathrm{H}-$ 1-benzopyranones of expected wide spectrum of potential bioresponses. Work along the expansion of such synthetic approach is now in progress.

\section{Experimental Section}

General Procedures. Melting points are uncorrected. IR spectra were recorded ( $\mathrm{KBr}$ disc) on a Pye Unicam SP-1000 spectrophotometer. ${ }^{1} \mathrm{H}-\mathrm{NMR}$ spectra were obtained on a Varian Gemini $200 \mathrm{MHz}$ spectrophoto-meter using TMS as an internal reference. Chemical shifts are expressed as $\delta(\mathrm{ppm})$. Mass spectra were recorded on a GCMS-QP 1000 Ex spectra mass spectrometer operating at $70 \mathrm{eV}$. Microanalytical data were performed by the Microanalytical Data Unit, Cairo University.

$N$-(2,2-Dicyanoethenyl)aminoacetonitrile (3). To a solution of aminoacetonitrile hydrogen sulphate (1) (100 mmol, $15.4 \mathrm{~g})$ in water $(60 \mathrm{~mL})$ containing sodium hydroxide (100 mmol, 4.0 $\mathrm{g})$, a solution of ethyl 2-cyano-3-ethoxypropenoate (2) (100 mmol, $12.2 \mathrm{~g})$ in EtOH (50 mL) was added portionwise. The reaction mixture was stirred for $2 \mathrm{~h}$ and left aside overnight at room temperature. The solid product that separated was collected by filtration and crystallized from ethanol to give $10.04 \mathrm{~g}(76 \%)$; mp $45-7^{\circ} \mathrm{C}$; IR (KBr) $3340(\mathrm{NH}), 2220,2216,2208 \mathrm{~cm}^{-1}(3 \mathrm{CN})$; ${ }^{1} \mathrm{H}-\mathrm{NMR}\left(\mathrm{DMSO}-\mathrm{d}_{6}\right) \delta 4.12\left(\mathrm{~s}, 2 \mathrm{H}, \mathrm{CH}_{2}\right), 7.32$ (s, 1H, CH), 9.11 (br s, 1H, NH, exchangeable); $\mathrm{m} / \mathrm{z}(\%) 132\left(\mathrm{M}^{+}, 18 \%\right)$; Anal. Calcd for $\mathrm{C}_{6} \mathrm{H}_{4} \mathrm{~N}_{4}$ : C, 54.5; H, 3.05; N, 42.4. Found: C, 54.4; $\mathrm{H}$, $3.0 ; \mathrm{N}, 42.2$.

$\boldsymbol{N}$-(2,2-Dicyanoethenyl)- $\boldsymbol{N}$-methylaminoacetonitrile (4). To a solution of 3 (20 mmol, $2.64 \mathrm{~g}$ ) in dry ether $(50 \mathrm{~mL})$, diazomethane $(20 \mathrm{mmol}, 0.84 \mathrm{~g})$ was added. The reaction mixture was stirred at ice-bath temperature $0-5{ }^{\circ} \mathrm{C}$ for $2 \mathrm{~h}$, then left aside overnight at $0{ }^{\circ} \mathrm{C}$. The mixture was evaporated under reduced pressure at $25{ }^{\circ} \mathrm{C}$, whereby the residue that precipitated was collected by filtration and crystallized from ether. Yield $2.07 \mathrm{~g}(71 \%)$; mp $49{ }^{\circ} \mathrm{C}$; IR (KBr) 2220, 2214, $2210 \mathrm{~cm}^{-1}(3 \mathrm{CN}) ;{ }^{1} \mathrm{H}-\mathrm{NMR}\left(\mathrm{DMSO}_{6}\right) \delta 2.12\left(\mathrm{~s}, 3 \mathrm{H}, \mathrm{CH}_{3}\right), 4.08\left(\mathrm{~s}, 2 \mathrm{H}, \mathrm{CH}_{2}\right), 7.18(\mathrm{~s}, 1 \mathrm{H}$, $\mathrm{CH}$ ); Anal. Calcd for $\mathrm{C}_{7} \mathrm{H}_{6} \mathrm{~N}_{4}$ : C, 57.5; $\mathrm{H}, 4.1 ; \mathrm{N}, 38.3$. Found: $\mathrm{C}, 57.4 ; \mathrm{H}, 4.1 ; \mathrm{N}, 38.2$.

Arylhydrazono- $N$-(2,2-dicyanoethenyl)- $N$-methylaminoacetonitriles $\quad 5 a, b . \quad$ (General Procedure). To a stirred solution of $4(5 \mathrm{mmol}, 0.73 \mathrm{~g})$ in ethanol $(50 \mathrm{~mL})$ containing NaOAc $(1.0 \mathrm{~g})$, the appropriate aryldiazonium chloride $(5 \mathrm{mmol})$ [prepared by adding $\mathrm{NaNO}_{2}\left(5 \mathrm{mmol}^{\text {, }}\right.$ $0.35 \mathrm{~g})$ to the appropriate primary aromatic amine $(5 \mathrm{mmol})$ in concentrated $\mathrm{HCl}(2 \mathrm{~mL})$ at $0-5$ ${ }^{\circ} \mathrm{C}$ while stirring] was added dropwise while cooling at $0-5{ }^{\circ} \mathrm{C}$ and stirring. The reaction mixture was left aside at room temperature for $3 \mathrm{~h}$, whereby the solid product that separated was collected by filtration, dried and crystallized from the appropriate solvent.

$N$-(2,2-Dicyanoethenyl)- $N$-methylphenylhydrazonoaminoacetonitriles (5a). $0.70 \mathrm{~g}$ (56\%); $\mathrm{mp} 105{ }^{\circ} \mathrm{C}(\mathrm{EtOH})$; IR (KBr) 2222, 2218, $2209 \mathrm{~cm}^{-1}$ (3CN); 1H-NMR (DMSO-d $)_{6} \delta 2.05$ (s, 3H, 
$\mathrm{CH}_{3}$ ), $7.16(\mathrm{~s}, 1 \mathrm{H}, \mathrm{CH}), 7.32-7.39$ (m, 5H, aromatic protons), 8.21 (s, 1H, NH, exchangeable); $\mathrm{m} / \mathrm{z}(\%) 250\left(\mathrm{M}^{+}, 22 \%\right)$; Anal. Calcd for $\mathrm{C}_{13} \mathrm{H}_{10} \mathrm{~N}_{6}$ : C, 62.4; H, 4.0; N, 33.6. Found: C, 62.2; $\mathrm{H}$, $4.0 ; \mathrm{N}, 33.5$.

p-Chlorophenylhydrazono- $N$-(2',2'-Dicyanoethenyl)- $N$-methylamino-acetonitriles (5b). 0.88 g (62\%); mp $121{ }^{\circ} \mathrm{C}(\mathrm{EtOH})$; IR (KBr) $3342(\mathrm{NH}), 2220,2218,2210 \mathrm{~cm}^{-1}(3 \mathrm{CN}) ;{ }^{1} \mathrm{H}-\mathrm{NMR}$ $\left(\mathrm{DMSO}_{-} \mathrm{d}_{6}\right) \delta 2.08\left(\mathrm{~s}, 3 \mathrm{H}, \mathrm{CH}_{3}\right), 7.14(\mathrm{~s}, 1 \mathrm{H}, \mathrm{CH}), 7.30-7.36(\mathrm{~m}, 4 \mathrm{H}$, aromatic protons), 8.52 (s, $1 \mathrm{H}, \mathrm{NH}$, exchangeable); Anal. Calcd for $\mathrm{C}_{13} \mathrm{H}_{9} \mathrm{ClN}_{6}$ : C, 54.8; H, 3.2; $\mathrm{Cl}, 12.45 ; \mathrm{N}, 29.5$. Found: C, 54.8; H, 3.0; Cl, 12.3; N, 29.5.

3-Amino-2,4-dicyano-1-methylpyrrole (6). A solution of 4 (5 mmol, $0.73 \mathrm{~g})$ in dry pyridine (30 $\mathrm{mL}$ ) was refluxed for $3 \mathrm{~h}$. The reaction mixture was cooled at room temperatures triturated with water, whereby the resulted solid product was collected by filtration, dried and crystallized from AcOH. Yield $0.39 \mathrm{~g} \mathrm{(54 \% );} \mathrm{mp} \mathrm{152-4}{ }^{\circ} \mathrm{C}(\mathrm{EtOH})$; IR (KBr) 3450-3380 $\left(\mathrm{NH}_{2}\right), 2218,2212 \mathrm{~cm}^{-1}$ $(2 \mathrm{CN}) ;{ }^{1} \mathrm{H}-\mathrm{NMR}\left(\mathrm{DMSO}-d_{6}\right) \delta 2.31\left(\mathrm{~s}, 3 \mathrm{H}, \mathrm{CH}_{3}\right), 6.33(\mathrm{~s}, 1 \mathrm{H}$, pyrrole 5-H), 10.71 (br s, 2H, $\mathrm{NH}_{2}$, exchangeable); Anal. Calcd for $\mathrm{C}_{7} \mathrm{H}_{6} \mathrm{~N}_{4}$ : C, 57.5; H, 4.1; N, 38.3. Found: C, 57.4; H, 4.0; N, 38.2.

Ethyl 3-amino-4-cyano-1-cyanomethylpyrrole-2-carboxylate (7). To a warm solution of 3 (5 mmol, $0.66 \mathrm{~g})$ in ethanol $(30 \mathrm{~mL})$, ethyl chloroacetate $(5 \mathrm{mmol}, 0.61 \mathrm{~g})$ in an aqueous $\mathrm{Na}_{2} \mathrm{CO}_{3}$ solution [ $\left(5 \mathrm{mmol}, 0.28 \mathrm{~g}\right.$ in $\left.\mathrm{H}_{2} \mathrm{O}(10 \mathrm{~mL})\right]$ was added. The reaction mixture was refluxed for 2 $\mathrm{h}$, left to cool at room temperature, poured onto cold water and neutralized with dilute $\mathrm{HCl}$. The solid product separated was filtered off, dried and crystallized from AcOH. Yield $0.60 \mathrm{~g}(55 \%)$; mp $142{ }^{\circ} \mathrm{C}(\mathrm{EtOH}) ; \mathrm{IR}(\mathrm{KBr}) 3445-3380\left(\mathrm{NH}_{2}\right), 2218,2210 \mathrm{~cm}^{-1}(2 \mathrm{CN}) ;{ }^{1} \mathrm{H}-\mathrm{NMR}$ (DMSO-d $\left.d_{6}\right) \delta$ $1.10\left(\mathrm{t}, 3 \mathrm{H}, \mathrm{CH}_{3}, J=7.2 \mathrm{~Hz}\right), 4.00\left(\mathrm{q}, 2 \mathrm{H}, \mathrm{CH}_{2}, J=7.2 \mathrm{~Hz}\right), 4.12\left(\mathrm{~s}, 2 \mathrm{H}, \mathrm{CH}_{2}\right), 6.31(\mathrm{~s}, 1 \mathrm{H}$, pyrrole 5-H), 10.62 (br s, 2H, $\mathrm{NH}_{2}$, exchangeable); $\mathrm{m} / \mathrm{z}(\%) 218\left(\mathrm{M}^{+}, 18 \%\right)$. Anal. Calcd for $\mathrm{C}_{10} \mathrm{H}_{10} \mathrm{~N}_{4} \mathrm{O}_{2}$ : C, 55.0; H, 4.6; N, 25.7. Found: C, 55.0; H, 4.5; N, 25.6.

1-Cyanomethyl-4-oxo-3-phenyl-1,2,3,4-tetrahydro-2-thioxopyrimidine (8). To a solution of 3 $(5 \mathrm{mmol}, 0.66 \mathrm{~g})$ in ethanol $(30 \mathrm{~mL})$, phenyl isothiocyanate $(5 \mathrm{mmol}, 0.67 \mathrm{~g})$ in aqueous solution of $\mathrm{NaOH}$ ( $5 \mathrm{mmol}, 0.20 \mathrm{~g}$ in $10 \mathrm{~mL} \mathrm{H}_{2} \mathrm{O}$ ) was added. The reaction mixture was refluxed for $3 \mathrm{~h}$, left to cool at room temperature, poured onto cold water and neutralized with dilute $\mathrm{HCl}$. The formed precipitate was filtered off, dried and crystallized from AcOH. Yield $0.86 \mathrm{~g} \mathrm{(64 \% );} \mathrm{mp}$ $163{ }^{\circ} \mathrm{C}(\mathrm{EtOH})$; IR (KBr) 2220, $2216(2 \mathrm{CN}), 1690(\mathrm{CO}) \mathrm{cm}^{-1} ; 1 \mathrm{H}-\mathrm{NMR}$ (DMSO-d 6$) \delta 4.08(\mathrm{~s}$, $\left.2 \mathrm{H}, \mathrm{CH}_{2}\right), 6.18(\mathrm{~s}, 1 \mathrm{H}$, pyrimidine $4-\mathrm{H}), 7.28-7.34(\mathrm{~m}, 5 \mathrm{H}$, aromatic protons); Anal. Calcd for $\mathrm{C}_{13} \mathrm{H}_{8} \mathrm{~N}_{4} \mathrm{OS}$ : C, 58.2; H, 3.0; N, 20.9; S, 11.95. Found: C, 58.0; H, 2.9; N, 20.7; S, 11.8 .

\section{5-Cyano-1-[1'-cyano-2'-dimethylaminoethenyl)-4-oxo-3-phenyl-1,2,3,4-tetrahydro-2-}

thioxopyrimidine (9). To a solution of 8 ( $5 \mathrm{mmol}, 1.34 \mathrm{~g})$ in dry dioxane $(30 \mathrm{~mL})$, dimethylformamide dimethylacetal (DMF-DMA) (5 mmol, $0.6 \mathrm{~g}$ ) was added. The reaction mixture was refluxed for $3 \mathrm{~h}$, evaporated in vacuo and triturated with ethanol. The resulting solid product was collected by filtration, dried and crystallized from dioxane. Yield $0.79 \mathrm{~g}(49 \%) ; \mathrm{mp} 175{ }^{\circ} \mathrm{C}$ (EtOH); IR (KBr) 2218, $2215(2 \mathrm{CN}), 1686 \mathrm{~cm}^{-1}(\mathrm{CO}) ;{ }^{1} \mathrm{H}-\mathrm{NMR}$ (DMSO-d $) \delta 2.86$ (s, 6H, $\left.2 \mathrm{CH}_{3}\right), 6.12(\mathrm{~s}, 1 \mathrm{H}$, pyrimidine $4-\mathrm{H}), 7.26-7.31$ (m, 5H, aromatic protons), $8.22(\mathrm{~s}, 1 \mathrm{H}, \mathrm{CH})$; Anal. Calcd for $\mathrm{C}_{16} \mathrm{H}_{13} \mathrm{~N}_{5} \mathrm{OS}$ : C, 59.4; H, 4.05; N, 21.65; S, 9.9. Found: C, 59.4; H, 3.8; N, 21.4; 
S, 9.8 .

1-[3'-Aminopyrazol-4'-yl]-5-cyano-4-oxo-3-phenyl-1,2,3,4-tetrahydro-2-thioxopyrimidine (10). A mixture of 9 ( $3 \mathrm{mmol}, 0.97 \mathrm{~g})$ and hydrazine hydrate $(3 \mathrm{mmol}, 0.97 \mathrm{~g})$ in ethanol $(30 \mathrm{~mL})$ was boiled under reflux for $30 \mathrm{~min}$, and then left aside at room temperature overnight. The mixture was poured onto cold water, whereby the solid product was filtered off and crystallized from EtOH. Yield $0.52 \mathrm{~g}(56 \%)$; mp $138{ }^{\circ} \mathrm{C}(\mathrm{EtOH})$; IR (KBr) 3445-3210 (NH and $\left.\mathrm{NH}_{2}\right), 2218$ $(\mathrm{CN}), 1690 \mathrm{~cm}^{-1}(\mathrm{CO}) ;{ }^{1} \mathrm{H}-\mathrm{NMR}\left(\mathrm{DMSO}_{6}\right) \delta 6.05(\mathrm{~s}, 1 \mathrm{H}$, pyrazole 5-H), $6.22(\mathrm{~s}, 1 \mathrm{H}$, pyrimidine 4-H), 7.26-7.31 (m, 5H, aromatic protons), 8.46 (s, 1H, NH, exchangeable), 9.62 (br s, $2 \mathrm{H}, \mathrm{NH}_{2}$, exchangeable); Anal. Calcd for $\mathrm{C}_{14} \mathrm{H}_{10} \mathrm{~N}_{6} \mathrm{OS}$ : C, 54.2; H, 3.25; N, 27.1; S, 10.3. Found: C, 54.0; H, 3.2; N, 26.9; S, 10.2.

$\mathrm{N}$-(2,2-Dicyanoethenyl)-2,2-dimethylaminoethenyl)aminoacetonitrile (11). To a solution of 3 $(10 \mathrm{mmol}, 1.32 \mathrm{~g})$ in dry dioxane $(30 \mathrm{~mL})$, dimethylformamide dimethylacetal (DMF-DMA) (10 mmol, $1.2 \mathrm{~g}$ ) was added. The reaction mixture was refluxed for $3 \mathrm{~h}$, evaporated in vacuo and triturated with ethanol. The resulting solid product was filtered, dried and crystallized from dioxane. Yield $1.01 \mathrm{~g}(54 \%) ; \mathrm{mp} 135^{\circ} \mathrm{C}(\mathrm{EtOH})$; IR (KBr) $3345(\mathrm{NH}), 2218,2216,2210 \mathrm{~cm}^{-1}$ $(3 \mathrm{CN}) ;{ }^{1} \mathrm{H}-\mathrm{NMR}\left(\mathrm{DMSO}-d_{6}\right) \delta 2.81\left(\mathrm{~s}, 6 \mathrm{H}, 2 \mathrm{CH}_{3}\right), 7.15(\mathrm{~d}, 1 \mathrm{H}, \mathrm{CH}), 8.16$ (br s, 1H, NH, exchangeable), 8.22 (s, $1 \mathrm{H}, \mathrm{CH})$; Anal. Calcd for $\mathrm{C}_{9} \mathrm{H}_{9} \mathrm{~N}_{5}$ : C, 57.7; H, 4.8; N, 37.4. Found: C, 57.6; H, 4.8; N, 37.3.

Reaction of 11 with heteroaryl amines 12, 14 and with C-nucleophile 16. (General procedure). To a solution of 11 (4 mmol, $0.75 \mathrm{~g}$ ) in glacial $\mathrm{AcOH}(30 \mathrm{~mL})$, the appropriate heteroaryl amine or C-nucleophile ( $4 \mathrm{mmol}$ ) was added. The reaction mixture was refluxed for 3 $\mathrm{h}$ and then left aside at room temperature overnight. The mixture was evaporated under reduced pressure, the residue was triturated with ethanol, whereby the solid precipitated so-formed was collected by filtration, dried and crystallized from the appropriate solvent.

3-(2,2-Dicyanoethenyl)amino-4H-pyrido[1,2-a]pyrimidin-4-one (13). Yield $0.39 \mathrm{~g}$ (41\%); mp $171{ }^{\circ} \mathrm{C}(\mathrm{EtOH})$; IR (KBr) 3420, $3415(\mathrm{NH}), 2220,2218(2 \mathrm{CN}), 1695 \mathrm{~cm}^{-1}$ (CO); ${ }^{1} \mathrm{H}-\mathrm{NMR}$ $\left(\right.$ DMSO-d $\left._{6}\right) \delta 7.21-7.32(\mathrm{~m}, 6 \mathrm{H}$, aromatic protons $\left.+\mathrm{CH})\right), 8.95(\mathrm{~s}, 1 \mathrm{H}, \mathrm{NH}$, exchangeable $) ; \mathrm{m} / \mathrm{z}$ (\%) $237\left(\mathrm{M}^{+}, 16 \%\right)$; Anal. Calcd for $\mathrm{C}_{12} \mathrm{H}_{7} \mathrm{~N}_{5} \mathrm{O}$ : C, 60.8; H, 3.0; N, 29.5. Found: C, 60.6; H, 2.9; N, 29.3.

6-(2,2-Dicyanoethenyl)amino-5H-thiazolo[1,2-a]pyrimidin-5-one (15). Yield $0.38 \mathrm{~g}$ (49\%); mp $193{ }^{\circ} \mathrm{C}(\mathrm{EtOH})$; IR (KBr) $3435(\mathrm{NH}), 2220,2216$ (2 CN), $1690 \mathrm{~cm}^{-1}$ (CO); ${ }^{1} \mathrm{H}-\mathrm{NMR}$ $\left(\mathrm{DMSO}_{6}\right) \delta 7.08(\mathrm{~s}, 1 \mathrm{H}$, pyrimidine $\mathrm{H}-4), 7.16(\mathrm{~d}, 2 \mathrm{H}$, thiazole $\mathrm{H}-4, \mathrm{H}-5), 7.28(\mathrm{~s}, 1 \mathrm{H}, \mathrm{CH})$, 8.92 (s, 1H, NH, exchangeable); Anal. Calcd for $\mathrm{C}_{10} \mathrm{H}_{5} \mathrm{~N}_{5} \mathrm{OS}: \mathrm{C}, 49.4 ; \mathrm{H}, 2.1 ; \mathrm{N}, 28.8 ; \mathrm{S}, 13.2$. Found: C, 49.3; H, 1.9; N, 28.6; S, 13.0.

3-(2,2-Dicyanoethenyl)amino-7,7-dimethyl-5-oxo-5,6,7,8-tetrahydro-2H-1-benzopyran-2one (17). Yield $0.49 \mathrm{~g}(43 \%)$; mp $210{ }^{\circ} \mathrm{C}(\mathrm{EtOH})$; IR (KBr) $3435(\mathrm{NH}), 2220,2216(2 \mathrm{CN})$, 1690, $1682 \mathrm{~cm}^{-1}(2 \mathrm{CO}) ;{ }^{1} \mathrm{H}-\mathrm{NMR}\left(\mathrm{DMSO}_{6}\right) \delta 1.24\left(\mathrm{~s}, 6 \mathrm{H}, 2 \mathrm{CH}_{3}\right), 2.48\left(\mathrm{~s}, 2 \mathrm{H}, \mathrm{CH}_{2}\right), 2.73(\mathrm{~s}$, $\left.2 \mathrm{H}, \mathrm{CH}_{2}\right), 7.58$ (s, 1H, H-4), $7.72(\mathrm{~d}, 1 \mathrm{H}, \mathrm{CHNH}), 10.52$ (d, 1H, NH, exchangeable); Anal. Calcd for $\mathrm{C}_{15} \mathrm{H}_{13} \mathrm{~N}_{3} \mathrm{O}_{3}$ : C, 63.6, H, 4.6; N, 14.8. Found: C, 63.5; H, 4.6; N, 14.7. 
Preparation of the free aminoheterocycles 18, 19 and 20 (General procedure). A mixture of the $\mathrm{N}$-(2,2-dicyanoethenyl)amino heterocyclic compound 13, 15 or 17 (2 mmol) and hydrazine hydrate $(2 \mathrm{mmol}, 0.1 \mathrm{~g})$ in ethanol $(15 \mathrm{~mL})$ was refluxed for $1 \mathrm{~h}$, and then left aside at room temperature overnight. The precipitated resulted was filtered off and crystallized from the appropriate solvent.

3-Amino-4H-pyrido[1,2-a]pyrimidin-4-one (18). Yield $0.17 \mathrm{~g}(52 \%)$; mp 178-180 ${ }^{\circ} \mathrm{C}(\mathrm{EtOH})$ (Lit. ${ }^{18} \mathrm{mp} \mathrm{180-2}{ }^{\circ} \mathrm{C}$ ); IR (KBr) 3450-3375 $\left(\mathrm{NH}_{2}\right), 1690 \mathrm{~cm}^{-1}$ (CO); ${ }^{1} \mathrm{H}-\mathrm{NMR}\left(\mathrm{DMSO}-d_{6}\right) \delta 5.57$ (br s, $2 \mathrm{H}, \mathrm{NH}_{2}$, exchangeable), 7.21-7.38 (m, 5H, aromatic protons); $\mathrm{m} / \mathrm{z}(\%) 161\left(\mathrm{M}^{+}, 14 \%\right)$; Anal. Calcd for $\mathrm{C}_{8} \mathrm{H}_{7} \mathrm{~N}_{3} \mathrm{O}$ : C, 59.6; H, 4.4; N, 26.1. Found: C, 59.5; H, 4.3; N, 25.9.

6-Amino-5H-thiazolo[3,2-a]pyrimidin-5-one (19). Yield $0.22 \mathrm{~g}(66 \%)$; mp 170-2 ${ }^{\circ} \mathrm{C}(\mathrm{EtOH})$; IR (KBr) 3454-3375 $\left(\mathrm{NH}_{2}\right), 1688 \mathrm{~cm}^{-1}(\mathrm{CO}) ;{ }^{1} \mathrm{H}-\mathrm{NMR}\left(\mathrm{DMSO}-d_{6}\right) \delta 5.14$ (br s, 2H, $\mathrm{NH}_{2}$, exchangeable), 7.15 (s, 1H, pyrimidine 4-H), 7.23 (d, 2H, thiazole H-4, H-5); Anal. Calcd for $\mathrm{C}_{6} \mathrm{H}_{5} \mathrm{~N}_{3} \mathrm{OS}$ : C, 43.1; H, 3.0; N, 25.1; S, 19.2. Found: C, 43.0; H, 2.8; N, 24.9; S, 19.0.

3-Amino-7,7-dimethyl-5-oxo-5,6,7,8-tetrahydro-2H-1-benzopyran-2-one (20). Yield $0.20 \mathrm{~g}$ (49\%); mp $149{ }^{\circ} \mathrm{C}(\mathrm{EtOH})$; IR (KBr) 3450-3364 $\left(\mathrm{NH}_{2}\right), 1695,1688 \mathrm{~cm}^{-1}$ (2 $\left.\mathrm{CO}\right)$; ${ }^{1} \mathrm{H}-\mathrm{NMR}$ $\left(\mathrm{DMSO}_{6}\right) \delta 1.26\left(\mathrm{~s}, 6 \mathrm{H}, 2 \mathrm{CH}_{3}\right), 2.44\left(\mathrm{~s}, 2 \mathrm{H}, \mathrm{CH}_{2}\right), 2.63\left(\mathrm{~s}, 2 \mathrm{H}, \mathrm{CH}_{2}\right), 5.35\left(\mathrm{br} \mathrm{s}, 2 \mathrm{H}, \mathrm{NH}_{2}\right.$, exchangeable), 7.40 (s, 1H, H-4); Anal. Calcd for $\mathrm{C}_{11} \mathrm{H}_{13} \mathrm{NO}_{3}: \mathrm{C}, 63.75 ; \mathrm{H}, 6.3 ; \mathrm{N}, 6.8$. Found: C, 63.6; H, 6.3; N, 6.6.

\section{References}

1. Erian, A. W. Chem. Rev. 1993, 93, 1991 and references cited therein.

2. Erian, A. W. J. Heterocycl. Chem. 2001, 38, 793 and references cited therein.

3. Erian, A. W.; Sherif, S. M. Tetrahedron 1999, 55, 7957 and references cited therein.

4. Sherif, S. M.; Erian, A. W. Heterocycles 1996, 43, 1085 and references cited therein.

5. Elnagdi, M. H.; Sherif, S. M.; Mohareb, R. M. Heterocycles 1987, 26, 496 and references cited therein.

6. Erian, A. W.; Issac, Y. A.; Sherif, S. M.; Mahmoud, F. F. J. Chem. Soc., Perkin Trans. 1 2000, 3686.

7. Erian, A. W.; Issac, Y. A.; Sherif, S. M. Z. Naturforsch. 2000, 55b, 127.

8. Erian, A. W.; Araki, V. F.; Aziz, S. I.; Sherif, S. M. Monatsh. Chem. 1999, 130, 661.

9. Sherif, S. M.; Youssef, M. M.; Mobark, K. M.; Abdel-Fattah, A. M. Tetrahedron 1993, 49, 9561.

10. Erian, A. W.; Sherif, S. M.; Alasser, A. A.; Elkholy, Y. M. Tetrahedron 1994, 50, 1877.

11. Ram, V. J.; Kushwaha, D. S.; Mishra, L. Indian J. Chem. 1989, 28B, 242.

12. Suguira, K.; Schind, A. F.; Schimd, M. M.; Brown F. G. Cancer Chemother. Rep. 1971, 3, 231.

13. Shishoo, C. J.; Jain, K. S. J. Heterocycl. Chem. 1992, 29, 883. 
14. Elslager, E. F.; Hess, C.; Johnson, J.; Ortwine, D.; Chien, V.; Werbel, L. M. J. Med. Chem. 1981, 24, 127.

15. Yao, H. C.; Resnick, P. J. J. Am. Chem. Soc. 1962, 84, 3514.

16. Yao, H. C. J. Org. Chem. 1964, 29, 2959.

17. Methcohn, O.; Tarnowski, B. Synthesis 1978, 56.

18. Selic, L.; Grdadolink, S. G.; Stanovnik, B. Heterocycles 1998, 49, 133. 